\title{
Improvising Security of HSZ Areas on Google Map Satellite Images of India via COTPCSD Mechanism
}

\author{
Sodadasi Sanjana Rachel \\ Dept. of Computer Science \& Engineering \\ Swarnandhra College of Engineering\&Technology \\ JNTUK, West Godavari, A.P,India
}

\author{
Dondapati Chittibabu \\ Dept. of Computer Science \& Engineering \\ Swarnandhra College of Engineering\&Technology \\ JNTUK, West Godavari, A.P, India
}

\begin{abstract}
The demand for Geospatail based services is improving dayto-day, due to its wide range of applications. But security is a major constraint for the satellite images with respect to Google Earth applications. In the recent times people are travelling easily to remote locations with a short span of time using the Google Earth application in the smart phones. In addition to that, it became a powerful tool for the antisocial elements in the society to execute terror attacks and bomb blasts at the right place. Especially India like country are prone to the terror and extremist attacks, which resulted in the greatest loss of thousands of lives. This problem can be addressed via., a novel Chaotic-One-Time-Password based Cryptic Satellite Data (COTPCSD) algorithm. The COTPCSD comprises of the Logistic map based chaotic image encryption and a One-Time-Password (OTP), which will strengthen the Quality-of-Security (QoS) for Google Earth Satellite data and reduces the Network Delay (ND) when compared to the existing Chaotic Multi-level Remote Sensing Data encryption (CMRSDE) algorithm. Our research outcomes show that the Overall Perfromance (OP) of COTPCSD is good compared to CMRSDE.
\end{abstract}

\section{General Terms}

Security, Algorithms, GIS.

\section{Keywords}

Satellite Data Security, Chaotic, Logistic encryption, COTPCSD, CMRSDE, Chaotic Multi-level Remote Sensing Data encryption, Chaotic-One-Time-Password based Cryptic Satellite Data, GIS, Remote Sensing, Matlab.

\section{INTRODUCTION}

With the ever-increasing demand of Geospatial data and its applicability taken a giant leap in the arena of Information and Communication Technology (ICT). Especially Geospatial applications involve many Geographic Information Systems (GIS) like smart cities, Google Earth, Global Positioning System, Geo-tagging, Disaster Management, Flood warning systems, Climate change, Environmental pollution, rainfall prediction, many location based services are more popular [1]. Exclusively Location-Based-Services through Google Earth (GE) satellite data plays a vital role in this digital age. Beside many usages with GE, finding a remote location and finding the shortest path between source and destination station applications is more popular. Also GE helps in finding the nearby important places like tourism spots, restaurants, government offices, rescue shelters, etc. are some of the fruitful works of the GE [2] [3]. Adjacent to that, GE became a robust device for the extremists or terrorists. With the help of GE map information, anti-social elements performed big bomblasts and terror operations all around the globe. Which in turn resulted in a big number of loss of lives and asserts. 26/11 Mumbai terror Attack is one such big instance, where militants used google maps for planned and executed perfectly, yield result in the loss of 171 deaths [4]. In another instance 42 people killed and 119 injured in the Hyderabad twin blasts in the 2007 year. Statstics were collected from various sources from the year 1984 to 2014. It is observed that on an average more than 40 people are killed by the terrorist every year in India. The terror attacks information is gathering of many newspaper articles information. This information provides for the past 30 years, approximately 59 incidents took place and more than 1889 deaths registered [AppendexI]. Any Country's own security is a core element for the development and prosperity of the nation. In the literature of the google map's security the country is further bifurcated into three categorical zones, they are High Prority Zone (HPZ), Average Prority Zone (APZ) and the Low Priority Zone (LPZ). Later cryptic algorithms were applied to the zones for their confidentiality.

To predict and prevent the terror attacks is still a million dollar question to the administers, scientists and defense forces. Previously proposed many studies were made and solutions proposed, but the QoS is high and ease of use is complex [5][6]. This work describes the privacy and confidentiality of the HPZ, APZ and LPZ based on the importance of the area in the society by maintaining reliability and fastness. The important contributions for the work are as follows: (1) a study and analysis terror attacks in Inida;(2) a Chatoic and OTP based Cryptic Satellite Data Algorithm; and (3) a novel performance metric by comprising security both QoS and ND; (4) an online working model where the COTPCSD mechanism is implemented and verified successfully. The organization of the paper is as follows. Background works described in Section 2 in the area of Chaotic logistic maps, Geospatail applications, and terrorist operations. The proposed solution COTPCSE algorithm is explained in Section 3. Online map server encryption experimentation and results discussed in Sections 4. The paper ended in section5 with a conclusion and future scope.

\section{RELATED WORKS}

The secure satellite data view on google maps is initiated by Rajesh Duvvuru et al., and proposed Chaotic Multilevel Remote Sensing Data Encryption (CMRSDE) Algorithm. They used double chaotic, based cryptic security of the satellite data and also categorized with various zones according to the geographical importance. In CMRSDE algorithm the ND takes more time with respect to the improvement in the QoS [7]. Whereas Xiaoqiang Zhang et al., proposed a Discrete Wavelet Transform (DWT) and Inverse Discrete Wavelet Transform based on two-dimensional logistic map [8]. Next to that Jin Liu and team addressed the problem of distributing the remote sensing image using Multirank encryption framework. The Multi-rank encryption mechanism depends on the usability level of the data for various users [9]. Recently Feifei Xin and group proposed a novel security mechanism using Terahertz wave for remote 
sensing images [10]. Nevertheless, more research has to perform on the Terahertz waves. Muhammad Usama used chaotic approach alone for the satellite data security [11]. Whereas google earth is all about integration of various NASA satellite images and other satellite repositories as Geographical Information System (GIS). Satellite data can broadly classify into spectral, temporal, Geometric, radiometric and spatial. Presently, Google satellite images comprise of mostly LandSat 8 satellite data. It contains 11 bands for various operations like coastal, aerosol studies and surface temperature, etc., with various spatial resolutions [12].

But the existing algorithms don't sustain the purpose of secure access to the HSZ. The proposed Chaotic-One-TimePassword based Cryptic Satellite Data (COTPCSD) algorithm is discussed in the next section and diagrammatically represented in figure 2 .

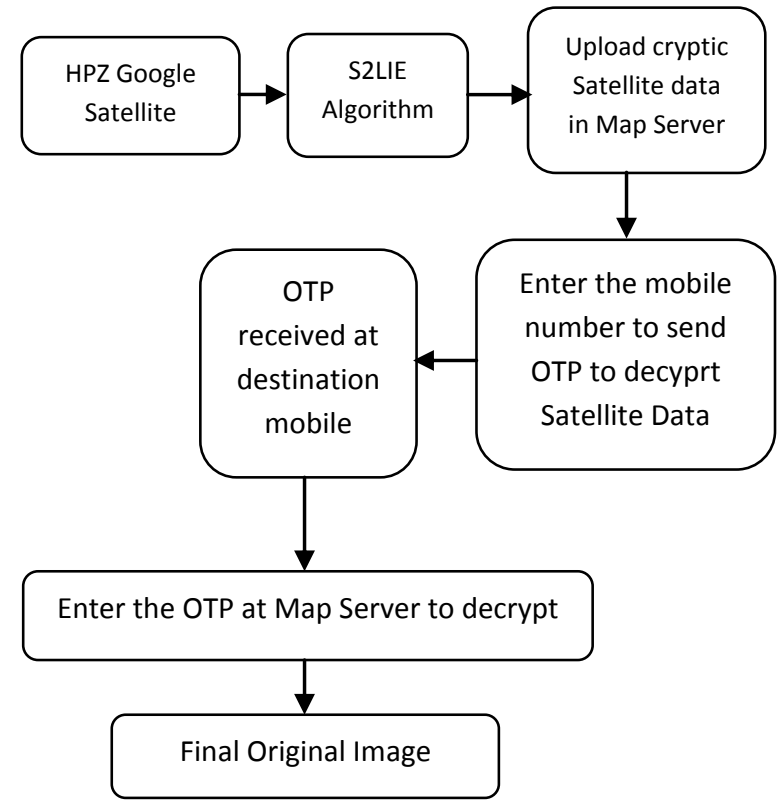

Fig 2: The data flow diagram of the COTPCSD mechanism.

The 2D Logistic Chaotic can be represented mathematically by the following equation.

$\mathrm{x}_{\mathrm{i}+1}=\mathrm{r}\left(3_{\mathrm{y}}+1\right) \mathrm{x}_{\mathrm{i}}\left(1-\mathrm{x}_{\mathrm{i}}\right)$ and

$\mathrm{y}_{\mathrm{i}+1}=\mathrm{r}\left(3_{\mathrm{x}}+1\right) \mathrm{y}_{\mathrm{i}}\left(1-\mathrm{y}_{\mathrm{i}}\right)$

Where Logistic $\left(x_{i+1}, y_{i+1}\right)$ is the spatial coordinates of $i$ ranges from $1,2,3 \ldots . \mathrm{n}$ and $\mathrm{r}$ is a chaotic behavior parameter with value 1.19 [13]

\section{CHAOTIC-ONE-TIME-PASSWORD BASED CRYPTIC SATELLITE DATA ALGORITHM}

\subsection{Notations and Assumptions}

The COTPCSD algorithm is designed and implemented with certain assumptions. The number of users access the google maps or google earth is assumed using Random Probability Distribution (RPD) function per day. It is precept that OTP generated will expires within 1 hour for one particular HSZ. The HSZ was identified according to area importance. The HSZ are as follows:

- Central Ministry Offices and Residential in Delhi .
- All Centrally funded Research Laboratories like the Baba Atomic Research Center (BARC), Council of Scientific \& Industrial Research (CSIR), Indian Space Research Organizations (ISRO), Defense Research Development Organization (DRDO) etc.

- State VIP Offices and Residences.

- Defense area in India.

- Popular pilgrim areas like Tirumala (Tirupati), Macca Majid (Hyderabad), St. Phelemon Church (Mysore), Varanasi temple etc.

\subsection{The Packet Model Adopted}

Various packet models were used in the architecture. Firstly the communication between client and server and Secondly the communication between Server and Mobile Phone via SMS. In general, the standard Peer-to-Point Extensible Authentication Protocol (PPP EAP) designated as RFC 2284 by Internet Engineering Task Force (IETF) adopted for the Mapserver-Mobile Network [14]. Whereas popular Hyper Text Transfer Protocol Secure (HTTPS) is inherited for the Client-Server communication [15].

\subsection{The COTPCSD Algorithm}

Step1: User access the Google Maps (GM) to access the HSZ.

Step2: Map Server raise and authentication interupt to the user.

Step3: Call Satellite 2D Logistic Image Encryption routine.

Step4: The send the KEY as OTP to the user using PPP EAP.

Step5:IF the sent OTP is received by the user,

$$
\text { GOTO Step6 }
$$

\section{ELSE Step7.}

Step6: Re-request for the OTP on the GM. GOTO Step4.

Step7: Enter the key to access the original HSZ map.

$$
\mathrm{IF}==\mathrm{KEY} \text {, then redirected to the original map }
$$

ELSE, GOTO Step1.

Step8: Stop.

\subsubsection{Satellite 2D Logistic Image Encryption (S2LIE) Algorithm}

Step1: Apply the Region-of-Interest (ROI) [16] on the HSZ of Google map images to perform segmentation.

Step2: Convert the segmented HSZ 3D images to 2D gray color images.

Step3: Perfrom the 2D Losgistic encryption on the 2D HSZ.

Step4: Replace the original image with the cryptic 2D HSZ. 


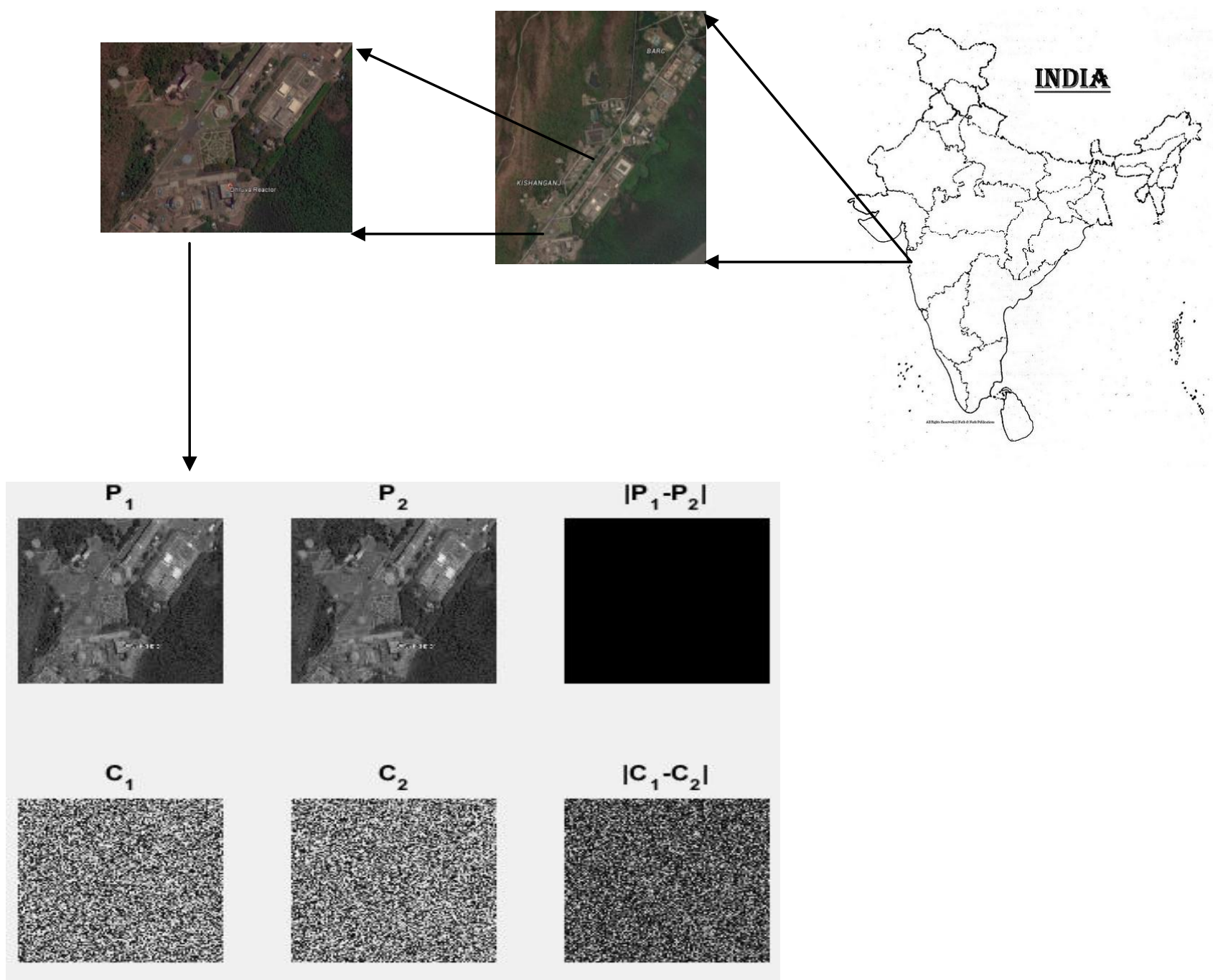

Fig 3: The S2LIE algorithm simulation results, where HSZ Study has been shown using India map.

\section{SIMULATIONS AND RESULTS}

\subsection{Simulation of S2LIE}

The HSZ satellite images are identified on the Google map India. The identified satellite images are segmented using ROI. The segmented images are in a 3 dimensional format. The 3D image encryption is a complex process and it takes more time and space to get encrypted. In order to get reduce the computation and beside maintaining the high security. The $3 \mathrm{D}$ images are converted into 2D images. By applying the rgb2gray() method; the 3D color images are converted into 2D gray scale images. The resulted HSZ grayscale satellite images are subjected to the 2D Logistic Encryption. The 2D Logistic encryption method contains Logistic-MDS(), Logistic-Permutations(), Logistic-Substitutions() and Logistic2D-image-cipher().

In this experiment, the $25 \mathrm{HSZ}$ satellite data were collected on Google map India images [17]. Among them Defense Research Development Organization Laboratory (DRDOL, Hyderabad), Baba Atomic Research Centre (BARC, Mumbai) and Indian Space Research Organization (ISRO, Banglore) are presented in the paper. The experimental results of S2LIE are shown in figure 3. The figure contains India map, segmented BARC image in color, segmented BARC image in grayscale and cryptic BARC Image. The step-bystep demonstration was shown below. Where as figure 4 and 5 the simulation results regarding cryptic DRDO and ISRO satellite data.

Table 1: Data used for the simulation

\begin{tabular}{|l|l|l|c|c|}
\hline \multicolumn{1}{|c|}{ HSZ } & \multicolumn{1}{|c|}{$\begin{array}{c}\text { Spatail } \\
\text { Coordinates }\end{array}$} & $\begin{array}{c}\text { Elevatio } \\
\mathbf{n}(\mathbf{f t})\end{array}$ & $\begin{array}{c}\text { Eye } \\
\text { alt } \\
\text { (ft) }\end{array}$ & $\begin{array}{c}\text { Date of } \\
\text { Image } \\
\text { Acquired }\end{array}$ \\
\hline DRDOL & $\begin{array}{c}17^{0} 20^{1} 20.60^{11} \mathrm{~N} \\
78^{0} 30^{1} 48.58^{11} \mathrm{E}\end{array}$ & 1720 & 2545 & $7 / 11 / 2014$ \\
\hline BARC & $\begin{array}{c}19^{0} 00^{1} 34.88^{11} \mathrm{~N} \\
72^{0} 55^{1} 34.96^{11} \mathrm{E}\end{array}$ & 36 & 3520 & $15 / 4 / 2015$ \\
\hline ISRO & $\begin{array}{c}12^{0} 57^{1} 35.02^{11} \mathrm{~N} \\
77^{0} 39^{1} 22.16^{11} \mathrm{E}\end{array}$ & 2933 & 3771 & $29 / 5 / 2015$ \\
& \multicolumn{2}{|l}{} & & \\
\hline
\end{tabular}




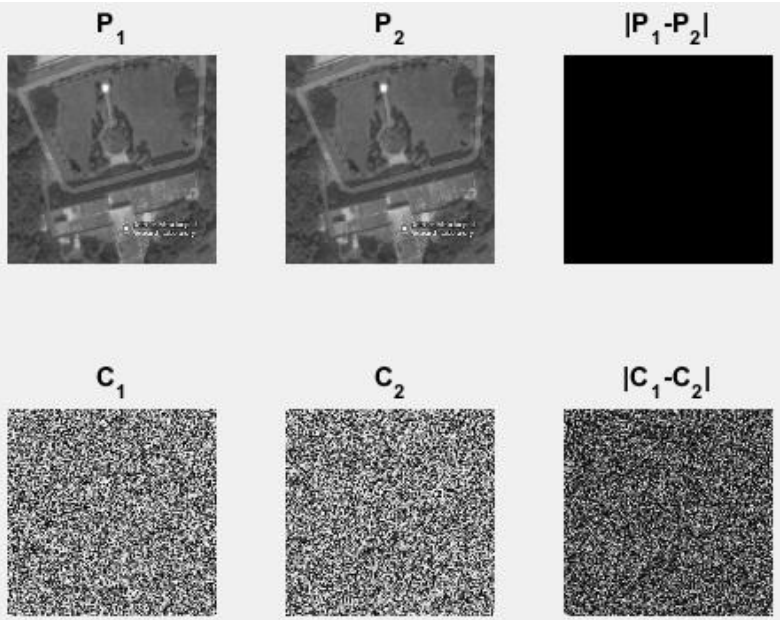

Fig 4: The S2LIE algorithm simulation results, where HSZ (DRDO Lab in Hyderabad).

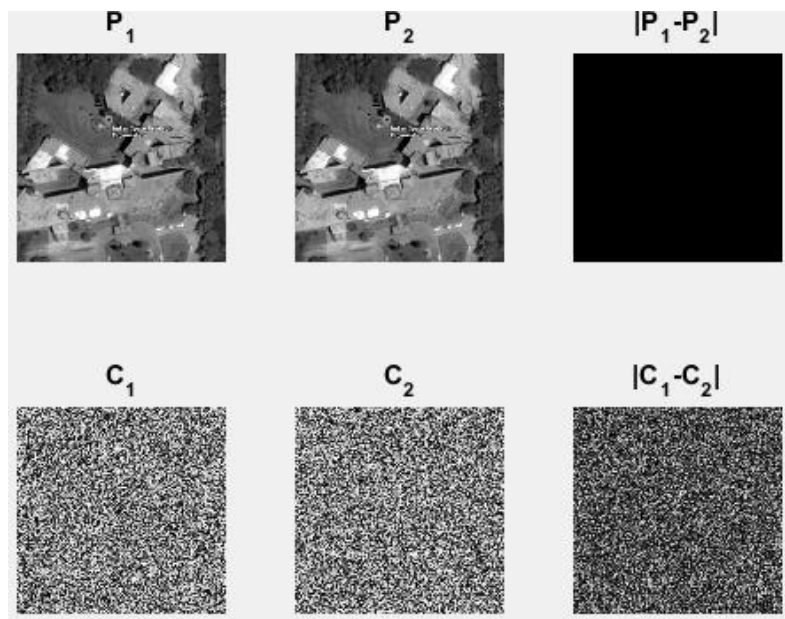

Fig 5: The S2LIE algorithm simulation results, where HSZ (ISRO in Banglore).

\subsection{Simulation of COTPCSD Algorithm}

The COTPCSD algorithm includes S2LIE simulation and PPP EAP implementation. To float the realtime PPP EAP implementation, a pack of 500 promotional, SMS is hired from the trusted third party from 'SMS Gateway Provider' company [18]. The simulation hosted on the Syamala Education Society (SES) website. The SES website is hosted on Linux-Apache-MySQL-PHP (LAMP) server and the SMS Interface was developed in PHP. Methods like curl_init(\$fullapiurl) and curl_setopt(\$ch, CURLOPT_RETURNTRANSFER, true) are used for secure password transformation. The SMS test is conducted for 42 mobile numbers and SMS is Sent Suessfully with various SMS delivered timely. Figure 6 shows the complete output of the COTPCSD, where the selected HSZ are encrypted using the 2D Logistic map.

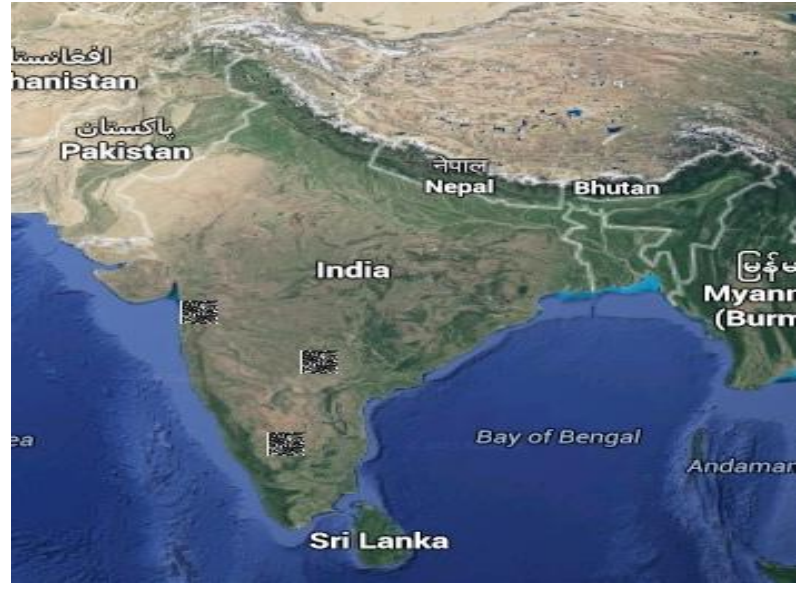

Fig 6: 2D Logistic map encryption performed on selected areas in India, Google satellite map.

\subsection{Impact of Encryption Time on Satellite images}

The COTPCSD compared with the CMRSDE based on the Encryption Time (ET). The CMRSDE has consumed more time than COTPCSD mechanism, because CMRSDE is an double encrypted mechanism, whereas COTPCSD is a single encryption algorithm. Figure 7 shows the comparative graph between the COTPCSD and CMRSDE based on ET.

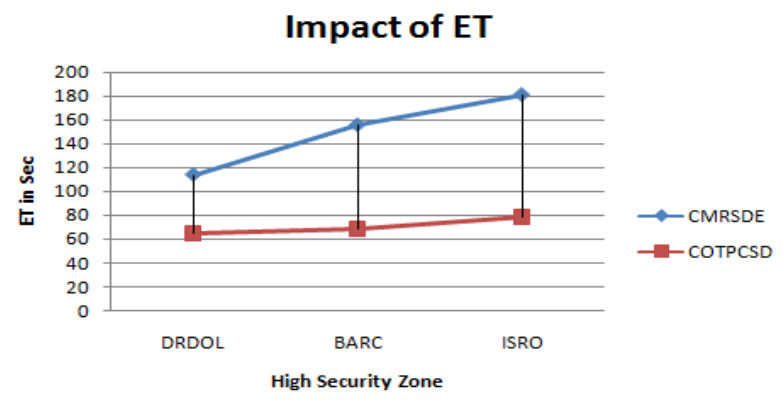

Fig 7: Impact of Encryption time on HSZ Satellite data.

\subsection{Impact on Overall Performance}

The Overall Performance (OP) can be calculated in the following the equation 2 .

$\mathrm{OP}=\operatorname{Logistic}\left(\mathrm{x}_{\mathrm{i}}, \mathrm{y}_{\mathrm{i}}\right)-\mathrm{ET}+\mathrm{SMSND}$

Where SMSND is the SMS network delay, which is assumed using RPD and the rest of the parameters are defined in the earlier sections.

\section{CONCLUSIONS AND FUTURE SCOPE}

Security to the satellite images is a key issue in the area of Geographic Information Systems. Beside maintaining the QoS and reducing the network delay is a big challenge to the researchers. This paper introduces the a novel COTPCSD, which uses the S2LIE Algorithm and PPP EAP for the secure and fast transmission of satellite data of HSZ, Google Satellite images. S2LIE uses the 2D Logistic Chaos map based encryption on the satellite data. PPP EAP is implemented by hiring the services of the SMS Gateway provider for the secure password transaction. The COTPCSD performance is comparatively better than CMRSDE, w.r.t. ET and OP. 
In future the 2D logistic map encryption can be replaced with more stronger chaotic map encryption for higher security.

\section{REFERENCES}

[1] Lu, Chang-Tien, Raimundo F. Dos Santos Jr, Lakshmi N. Sripada, and Yufeng Kou. "Advances in GML for geospatial applications." Geoinformatica 11, no. 1 (2007): 131-157.

[2] Schiller, Jochen, and Agnès Voisard, eds. Location-based services. Elsevier, 2004

[3] Wilson, Matthew W. "Location-based services, conspicuous mobility, and the location-aware future." Geoforum 43.6 (2012): 1266-1275.

[4] Azad, Sarita, and Arvind Gupta. "A quantitative assessment on 26/11 Mumbai attack using social network analysis." Journal of Terrorism Research 2, no. 2 (2011).

[5] Rajesh Duvvuru, S.K Singh, K. Gowri Sankar and S. Deepthi. "Prediction and Analysis of Bomb Blasts in India." In International Conference on Communication and Computing (ICC-2015). 114-120, 2014.

[6] Booth, Ken, and Tim Dunne. Worlds in collision: terror and the future of global order. Palgrave Macmillan, 2002.

[7] Duvvuru, Rajesh, P. Jagadeeswara Rao, and Gudikandhula Narasimha Rao. "Multi-Level Chaos Based Encryption Mechanism to Enhance Security of High Security Zone Areas on Google Map Satellite Images of India." International Journal of Applied Engineering Research 10.3 (2015): 8059-8072.

[8] Zhang, Xiaoqiang, Guiliang Zhu, and Shilong Ma. "Remote-sensing image encryption in hybrid domains." Optics Communications 285, no. 7 (2012): 1736-1743.

[9] Liu, Jin, Zhengquan Xu, Jing Sun, Xiaojun Liu, and Zhe Wu. "Network distribution of remote sensing images based on multi-rank security." InComputer Sciences and Convergence Information Technology, 2009. ICCIT'09.
Fourth International Conference on, pp. 1101-1104. IEEE, 2009.

[10] Xin, Feifei, Hongyan Su, and Yong Xiao. "Terahertz imaging system for remote sensing and security applications." In Antennas and Propagation (APCAP), 2014 3rd Asia-Pacific Conference on, pp. 1335-1338. IEEE, 2014.

[11] Usama, Muhammad, Muhammad Khurram Khan, Khaled Alghathbar, and Changhoon Lee. "Chaos-based secure satellite imagery cryptosystem."Computers \& Mathematics with Applications 60, no. 2 (2010): 326337.

[12] Roy, David P., M. A. Wulder, T. R. Loveland, C. E. Woodcock, R. G. Allen, M. C. Anderson, D. Helder et al. "Landsat-8: Science and product vision for terrestrial global change research." Remote Sensing of Environment 145 (2014): 154-172.

[13] Wu, Yue, Gelan Yang, Huixia Jin, and Joseph P. Noonan. "Image encryption using the two-dimensional logistic chaotic map." Journal of Electronic Imaging21, no. 1 (2012): 013014-1.

[14] Blunk, Larry J. "PPP extensible authentication protocol (EAP)." (1998).

[15] Callegati, Franco, Walter Cerroni, and Marco Ramilli. "Man-in-the-Middle Attack to the HTTPS Protocol." IEEE Security \& Privacy 1 (2009): 78-81.

[16] Liu, Lijie, and Guoliang Fan. "A new JPEG2000 regionof-interest image coding method: partial significant bitplanes shift." Signal Processing Letters, IEEE 10, no. 2 (2003): 35-38.

[17] https://www.google.co.in/maps/@20.9857003,82.752630 $5,2842363 \mathrm{~m} /$ data $=! 3 \mathrm{~m} 1 ! 1 \mathrm{e} 3$ ?hl=te (Accessed on $05 / 05 / 2015$

[18] http://smsgatewayprovider.com/ (Accessed on $21 / 05 / 2015)$

\section{APPENDIX}

Table 2: List of Terror attacks in India and resulted deaths

\begin{tabular}{|c|l|l|c|c|}
\hline DATE & \multicolumn{1}{|c|}{ INCIDENT } & \multicolumn{1}{|c|}{ PLACE } & DEADLINESS & HURT \\
\hline $08 / 02 / 1984$ & Meenambakkam Bomb Blast & Tamil Nadu & 30 & 25 \\
\hline $07 / 07 / 1987$ & Punjab Killings & Punjab & 36 & 60 \\
\hline $15 / 06 / 1991$ & Punjab Killings & Punjab & 90 & 200 \\
\hline $03 / 12 / 1993$ & Bombay Bomb Blasts & Mumbai & 259 & 713 \\
\hline $30 / 12 / 1996$ & Bramhaputra Mail Train Bomb Blast & & 33 & 150 \\
\hline $14 / 02 / 1998$ & Coimbatore Bomb Blast & Tamil Nadu & 58 & $200+$ \\
\hline $22 / 12 / 2000$ & Terrorist Attack On Red Fort & Delhi & 3 & 14 \\
\hline $10 / 01 / 2001$ & Jammu and Kashmir Legislative Assembly Attack & Kashmir & 38 & 0 \\
\hline $13 / 12 / 2001$ & Indian Parliament Attack In New Delhi & Delhi & & 7 \\
\hline
\end{tabular}


International Journal of Computer Applications (0975 - 8887)

Volume 128 - No.13, October 2015

\begin{tabular}{|c|c|c|c|c|}
\hline $13 / 05 / 2002$ & Janupur Train Crash & Uttar Pradesh & 12 & 80 \\
\hline $12 / 06 / 2002$ & Mumbai Bus Bomb Blasts & Mumbai & 2 & 14 \\
\hline $21 / 12 / 2002$ & Kurnool Train Crash & Andhra Pradesh & 20 & 80 \\
\hline $09 / 10 / 2002$ & Rajiganji Train Disaster & Bihar & 130 & 300 \\
\hline 24/09/2002 & $\begin{array}{l}\text { Terrorists Attack The Akshadharam Temple in } \\
\text { Gujarat }\end{array}$ & Gujarat & 31 & 0 \\
\hline $27 / 01 / 2003$ & Mumbai Bomb Blasts & Mumbai & 1 & 0 \\
\hline $13 / 03 / 2003$ & Mumbai Train Bomb Blasts & Mumbai & 11 & 0 \\
\hline $28 / 07 / 2003$ & Mumbai Bus Bomb Blasts & Mumbai & 4 & 32 \\
\hline $25 / 08 / 2003$ & Mumbai Bomb Blasts & Mumbai & 52 & 0 \\
\hline $15 / 08 / 2004$ & Dhemaji School Bomb Blast & Assam & 18 & 40 \\
\hline $28 / 07 / 2005$ & Janupur Train Bomb Blast & Uttar Pradesh & 13 & 50 \\
\hline $29 / 10 / 2005$ & $\begin{array}{l}\text { Three Powerful Serial Blasts In New Delhi At } \\
\text { Different Places }\end{array}$ & Delhi & 70 & 250 \\
\hline 03/07/2006 & Varanasi Bomb Blasts & Varanasi & 21 & \\
\hline $07 / 11 / 2006$ & Mumbai Train Bomb Blasts & Mumbai & 209 & 500 \\
\hline $09 / 08 / 2006$ & Malegaon Bomb Blasts & Maharashtra & 37 & 125 \\
\hline $18 / 02 / 2007$ & Samjhauta Express Bomb Blast & Harayana & 68 & 0 \\
\hline $18 / 05 / 2007$ & Mecca Masjid Bomb Blast & Hyderabad & 13 & 0 \\
\hline DATE & INCIDENT & PLACE & DEADLINESS & HURT \\
\hline $25 / 08 / 2007$ & Hyderabad Bomb Blast & Hyderabad & 42 & 0 \\
\hline $10 / 11 / 2007$ & $\begin{array}{l}\text { One Blast At A Shrine Of A Sufi Muslim Saint In The } \\
\text { Town Of Ajmer }\end{array}$ & Rajasthan & 3 & 0 \\
\hline $14 / 10 / 2007$ & $\begin{array}{l}\text { One Blast In A Movie Theater In The Town Of } \\
\text { Ludhiana Of Muslim Holy Day Of Eid-Ul-Fitr }\end{array}$ & Ludhiana & 6 & 0 \\
\hline $24 / 11 / 2007$ & $\begin{array}{l}\text { A Series Of Near-Simultaneous Explosions At } \\
\text { Courthouse Complexes In The Cities Of Lucknow, } \\
\text { Varanasi and Faizabad }\end{array}$ & Uttar Pradesh & 16 & 70 \\
\hline $01 / 01 / 2008$ & $\begin{array}{l}\text { Terror Attack on CRPF Camp In Rampur, Uttar } \\
\text { Pradesh By Lashkar-E-Taiba }\end{array}$ & Uttar Pradesh & 8 & 5 \\
\hline $13 / 05 / 2008$ & Jaipur Bomb Blasts & Jaipur & 63 & 200 \\
\hline $25 / 07 / 2008$ & Banglore Serial Bomb Blast & Banglore & 2 & 20 \\
\hline $26 / 08 / 2008$ & Ahmedabad Blasts & Gujarat & 29 & 110 \\
\hline $13 / 09 / 2008$ & Delhi Bomb Blasts & Delhi & 33 & 130 \\
\hline $27 / 09 / 2008$ & Western India Bomb Blasts & Maharashtra & 10 & 80 \\
\hline $10 / 01 / 2008$ & Agartala Bomb Blasts & Agartala & 8 & 100 \\
\hline
\end{tabular}




\begin{tabular}{|c|c|c|c|c|}
\hline $21 / 10 / 2008$ & Imphal Bomb Blasts & Imphal & 17 & 40 \\
\hline $30 / 10 / 2008$ & Assam Bomb Blasts & Assam & 77 & 300 \\
\hline $26 / 11 / 2008$ & Mumbai Attacks & Mumbai & 171 & 239 \\
\hline $01 / 01 / 2009$ & Guwahati Bomb Blasts & Assam & 6 & 67 \\
\hline $04 / 06 / 2009$ & Assam Bomb Blasts & Assam & 7 & 62 \\
\hline $13 / 02 / 2010$ & Pune Bomb Blasts & Pune & 17 & 60 \\
\hline $12 / 07 / 2010$ & Varanasi Bomb Blasts & Varanasi & 1 & 20 \\
\hline $13 / 07 / 2011$ & Mumbai Bomb Blasts & Mumbai & 26 & 130 \\
\hline 09/07/2011 & Delhi Bomb Blasts & Delhi & 19 & 76 \\
\hline $13 / 02 / 2012$ & Attacks On Israeli Diplomats & Delhi & 0 & 4 \\
\hline $08 / 01 / 2012$ & Pune Bomb Blasts & Pune & 0 & 1 \\
\hline $21 / 02 / 2013$ & Hyderabad Bomb Blast & Hyderabad & 16 & 119 \\
\hline $13 / 03 / 2013$ & Srinagar Attack & $\begin{array}{l}\text { Jammu and } \\
\text { Kashmir }\end{array}$ & 7 & 10 \\
\hline $17 / 04 / 2013$ & Banglore Blast & Bengaluru & 0 & 16 \\
\hline $25 / 05 / 2013$ & Naxal Attack In Darbha Valley & Chhattisgarh & 28 & 32 \\
\hline DATE & INCIDENT & PLACE & DEADLINESS & HURT \\
\hline $24 / 06 / 2013$ & Srinagar Attack & $\begin{array}{l}\text { Jammu and } \\
\text { Kashmir }\end{array}$ & 8 & 19 \\
\hline $07 / 07 / 2013$ & Maoist Attack In Dumka & Chhattisgarh & 5 & 0 \\
\hline $07 / 07 / 2013$ & Bodh Gaya Bomb Blasts & Bihar & 0 & 5 \\
\hline $27 / 10 / 2013$ & Patna Bomb Blasts & Bihar & 5 & 66 \\
\hline $25 / 04 / 2014$ & Blast In Jharkhand & Jharkhand & 8 & 45 \\
\hline $28 / 04 / 2014$ & Blast In Budgam District & $\begin{array}{l}\text { Jammu and } \\
\text { Kashmir }\end{array}$ & 0 & 18 \\
\hline $05 / 01 / 2014$ & Chennai Train Bomb Blast & Tamil Nadu & 1 & 14 \\
\hline $05 / 12 / 2014$ & Maoist Blast In Gadchiroli District & Jharkhand & 7 & 2 \\
\hline $28 / 12 / 2014$ & Bomb Blast At Church Street Banglore & Bengaluru & 1 & 5 \\
\hline $20 / 03 / 2015$ & Jammu Attack & $\begin{array}{l}\text { Jammu and } \\
\text { Kashmir }\end{array}$ & 6 & 10 \\
\hline $27 / 07 / 2015$ & Gurdaspur Attack In Dina Nagar, Gurdaspur District & Punjab & 10 & 15 \\
\hline
\end{tabular}

